\title{
Determination of the best model for estimating heritability of economic traits and their genetic and phenotypic trends in Iranian native fowl
}

\author{
Mona Salehinasab', Saeed Zerehdaran ${ }^{\square}$, Mokhtar Ali Abbasi ${ }^{\square}$, Sadegh Alijani ${ }^{\square}$ and \\ Saeed Hassani ${ }^{\square}$
}

${ }^{1}$ Gorgan University of Agricultural Sciences and Natural Resources, Gorgan, Iran, ${ }^{2}$ Animal Research Institute, Karaj, Iran, ${ }^{3}$ Tabriz University, Tabriz, Iran

\section{Abstract}

The objective of the present study was to estimate the heritability and to assess the existence of maternal effects for economic traits in Iranian native fowl. Variance components were estimated for body weight at hatch $\left(\mathrm{BW}_{0}\right), 8\left(\mathrm{BW}_{8}\right)$ and $12\left(\mathrm{BW}_{12}\right)$ weeks of age, age at sexual maturity and weight at sexual maturity, egg number and average egg weight at 28th, 30th and 32nd weeks using restricted maximum likelihood method and six animal models. The best model was determined using the Akaike information criterion for each trait. For age at sexual maturity, the basic model consisting of direct genetic effects was superior. For weight at sexual maturity and egg number, a model consisting of maternal permanent environmental effects in addition to direct genetic effects was the best. For average egg weight at 28th, 30th and 32nd weeks, the model with direct and maternal genetic effects assuming no covariance between them was the best. For $\mathrm{BW}_{0^{\prime}} \mathrm{BW}_{8}$ and $\mathrm{BW}_{12^{\prime}}$ the model including maternal genetic and permanent environmental effects in addition to direct genetic effects was the most appropriate. The estimates of direct heritability ranged from $0.05\left(\mathrm{BW}_{0}\right)$ to 0.35 (weight at sexual maturity). Direct genetic variance and heritability were overestimated if maternal effects were ignored in the statistical model for all traits except ASM. The results indicated that the evaluation of direct and maternal genetic and phenotypic trends based on the best model for each trait was carried out. Maternal genetic trends for $\mathrm{BW}_{0}, \mathrm{BW}_{8}, \mathrm{BW}_{12}$ and average egg weight at 28th, 30th and 32nd weeks were significantly positive. Present results indicated favourable effects of the performed breeding program for all traits except $\mathrm{BW}_{0^{\prime}}$ during generations. 
Keywords: native fowl, restricted maximum likelihood, economic traits, maternal genetic trend, direct heritability, maternal effects Abbreviations: AIC: Akaike information criterion, $\mathrm{ASM}$ : age at sexual maturity, $\mathrm{BW}_{0}$ : body weight at hatch,
$\mathrm{BW}_{8}$ : body weight at eight weeks of age, $\mathrm{BW}_{12}$ : body weight at 12 weeks of age, EN: egg number,
EW: average egg weight at 28 th, 30 th and $32 \mathrm{nd}$ weeks, WSM: weight at sexual maturity

\section{Introduction}

Indigenous chickens, despite their low growth rate and egg production, are generally better in disease resistance and could maintain higher level of performance under poor nutrition and high environmental temperatures compared to commercial strains under village systems (Horst 1989). Iranian indigenous chickens are meat-cum-egg type. Breeding of native fowl is important for small farmers to produce more income and also to conserve genetic variation of native breeds (Emamgholi Begli et al. 2010). Kiani-Manesh (2000) showed that age at sexual maturity, egg number, egg weight and body weight at eight weeks of age are the most important traits for improving the economic efficiency of Iranian native fowl. One of the effective ways for improving production performance is genetic improvement in these populations. Thus, the estimation of variance components, genetic parameters and breeding values is very important.

Several studies have been conducted on estimating direct heritabilities for economic traits with no attention to the existence of maternal effects in native fowl (Sang et al. 2006, Kamali et al. 2007). But only a few estimates are available for maternal genetic and permanent environmental effects (Norris \& Ngambi 2006, Haunshi et al. 2012, Ghorbani et al. 2012). So far, no estimate is available for trends based on the best model for economic traits in these populations.

Maternal effects play an important role in development of the economic traits. These effects can be caused by genetic or environmental differences between mothers or by the combination of the genetic and environmental differences (Grosso et al. 2010). Meyer (1997) suggested that including maternal effects in the analysis reduces the bias in the estimate of genetic variance. Maternal effects in birds are different from those of mammals, because any maternal effect on chicks, incubated artificially, must be the residual effect of dam reflected in egg characteristics at laying (Saatci et al. 2006).

The objective of the current study was to determine the importance of the maternal additive genetic and permanent environmental effects to estimate heritabilities for economic traits in Iranian native fowl. In addition, the breeding program performed for the studied traits is evaluated based on genetic and phenotypic trends obtained from the best model.

\section{Material and methods}

The records of the economic traits were collected from native fowl of the Isfahan breeding centre. Isfahan province is located in the centre of Iran and its climate is dry and hot with an average temperature of more than $40^{\circ} \mathrm{C}$ and a humidity of lower than $25 \%$ in the summer. Native fowl of this area have a reasonable production performance because of their 
adaptation to dry and high temperature conditions. The Isfahan native fowl breeding centre has been established in 1980. This centre started its activity by collecting native fowl from far rural areas. Native fowl were selected based on their phenotypic characteristics. The base population was generated from 200 native fowl (100 of each sex), and the first generation was produced by random mating of the base population. From the first generation, body weight at 12 weeks of age $\left(\mathrm{BW}_{12}\right)$, age at sexual maturity (ASM) and weight at sexual maturity (WSM), number of eggs during the first 12 weeks of laying period (EN) and average egg weight at 28th, 30th and 32nd weeks (EW) were collected. Birds were selected as parents of the next generation in two steps. In the first step, females and males were selected based on their BW $_{12}$. After 20 weeks of age, hens were transferred into individual cages and their egg production was recorded for 12 weeks. In the second step, hens were selected based on ASM, WSM, EN and EW, and cocks were selected based on the performance of their sisters. An average selection proportion of about $40 \%$ for hens and $5 \%$ for cocks was applied in each generation, in which 800 hens and 100 cocks were selected to produce the next generation. In total, 14 generations have been generated in Isfahan breeding centre. The economic traits including $\mathrm{BW}_{0^{\prime}} \mathrm{BW}_{8^{\prime}} \mathrm{BW}_{12^{\prime}} \mathrm{ASM}$, WSM, EN and EW were collected and used in the analyses.

\section{Statistical analyses}

The Anderson-Darling test for normality was used to explore the distribution of all data (Anderson \& Darling 1952). The GLM procedure of the SAS 9.1 software (SAS Institute Inc., Cary, NC, USA) was applied to determine the significance of fixed effects using the F-test.

The variance components and the heritabilities were estimated by different animal models using the restricted maximum likelihood method. The analyses were done using the ASREML software (Gilmour et al. 2000).

Fixed effects were a combination of generation and hatch ( 45 levels), birth year ( 13 levels) and sex ( 2 levels). The effect of days of production was considered as covariate only for egg number.

The following six univariate animal models were used:

$$
\begin{array}{lll}
\text { Model 1: } & y=X b+Z_{1} a+e & \\
\text { Model 2: } & y=X b+Z_{1} a+W c+e & \\
\text { Model 3: } & y=X b+Z_{1} a+Z_{2} m+e & \operatorname{Cov}_{a m}=0 \\
\text { Model 4: } & y=X b+Z_{1} a+Z_{2} m+e & \operatorname{Cov}_{a m} \neq 0 \\
\text { Model 5: } & y=X b+Z_{1} a+Z_{2} m+W c+e & \operatorname{Cov}_{a m}=0 \\
\text { Model 6: } & y=X b+Z_{1} a+Z_{2} m+W c+e & \operatorname{Cov}_{a m} \neq 0
\end{array}
$$

In these models, $\boldsymbol{y}$ is the vector of observations, $\boldsymbol{b}$ is the vector of fixed effects, $\boldsymbol{a}$ is the vector of random direct additive genetic effects, $m$ is the vector of random maternal additive genetic effects, $\boldsymbol{c}$ is the vector of random maternal permanent environmental effects, $\boldsymbol{e}$ is the vector of random residual effects, and $X, Z_{1}, Z_{2^{\prime}}$ and $W$ are the incidence matrices relating the observations to the fixed, direct genetic, maternal additive genetic and maternal permanent environmental effects, respectively. $\operatorname{Cov}_{\mathrm{am}}$ indicates whether covariance between direct and maternal genetic effects was considered. Models were compared using the Akaike information criterion (AIC) to find the best model for each trait. AIC values are a function of log-likelihood values and the number of free parameters estimated by a model to determine 
the goodness-of-fit of the model and the effectiveness of adding extra parameters (Akaike 1974). Log-likelihood values produced by the ASREML software (and AIC values calculated from these outputs) were used as a guide to compare the fit of each model.

Finally, the genetic, phenotypic and environmental trends were evaluated based on the best model for each trait. These values were obtained as the regression coefficients of the direct or maternal breeding values, phenotypic and environmental values on generations.

\section{Results and discussion}

Descriptive statistics including significance levels for testing of fixed effects and $P$-values of testing for normality for all traits are presented in Table 1.

Table 1

Descriptive statistics, test of significance of fixed effects and normality for studied traits

\begin{tabular}{lcrrrrrr}
\hline Trait & Number & Mean & CV & Birth year $^{2}$ & Sex $^{1}$ & GH $^{2}$ & $P-A D$ \\
\hline BW $_{0^{\prime}}$ g & 51521 & 37.72 & 9.06 & $* * *$ & $0.55^{* * *}$ & $* * *$ & 0.27 \\
BW $_{8^{\prime}}$ g & 45517 & 842.58 & 18.46 & $* * *$ & $147.48^{* * *}$ & $* * *$ & 0.18 \\
BW $_{12^{\prime}}$ g & 45864 & 1381.19 & 17.10 & $* * *$ & $319.62^{* * *}$ & $* * *$ & 0.25 \\
ASM, day $_{\text {WSM, day }}^{19745}$ & 178.94 & 8.72 & $* * *$ & - & $* * *$ & 0.33 \\
EN, number & 20000 & 1883.38 & 10.49 & $* * *$ & - & $* * *$ & 0.14 \\
EW, g & 17606 & 47.05 & 25.87 & $* * *$ & - & $* * *$ & 0.47 \\
\hline
\end{tabular}

'Difference of male and female for each trait was shown as sex effect, ${ }^{2}$ Because of large effects of birth year and $\mathrm{GH}$, only the significance level was shown, ${ }^{* * *} P<0.001, \mathrm{CV}$ : coefficient of variation, $\mathrm{GH}$ : combination of generation and hatch effects, P-AD: $P$-value of Anderson-Darling test

All traits were normally distributed and the effect of sex, birth year and the combination of generation and hatch were significant for all traits. The mean values of $\mathrm{BW}_{0^{\prime}} \mathrm{BW}_{8^{\prime}} \mathrm{BW}_{12^{\prime}}$, and WSM were $37.72,842.58,1381.19$ and $1883.38 \mathrm{~g}$, respectively and the mean values of ASM, EN and EW were 178.94 days, 47.05 eggs and $48.91 \mathrm{~g}$, respectively.

The best model for each trait, direct and maternal heritabilities and the proportion of maternal permanent environmental effects to phenotypic variance are shown in Table 2.

Table 2

The best models, direct and maternal genetic heritability estimates, the proportion of maternal permanent environment to phenotypic variance $\left(c^{2}\right)$ and direct heritability obtained from model 1 with their standard errors for studied traits

\begin{tabular}{lccccc}
\hline Trait & Best model & Direct heritability & Maternal heritability & Phenotypic variance & $\begin{array}{c}\text { Direct heritability } \\
\text { (Model } 1 \text { ) }\end{array}$ \\
\hline BW $_{0}$ & 5 & $0.05 \pm 0.01$ & $0.30 \pm 0.02$ & $0.25 \pm 0.02$ & $0.56 \pm 0.00$ \\
BW $_{8}$ & 5 & $0.24 \pm 0.01$ & $0.01 \pm 0.01$ & $0.04 \pm 0.01$ & $0.33 \pm 0.01$ \\
BW $_{12}$ & 5 & $0.17 \pm 0.01$ & $0.02 \pm 0.01$ & $0.03 \pm 0.01$ & $0.26 \pm 0.01$ \\
ASM & 1 & $0.31 \pm 0.02$ & - & - & $0.31 \pm 0.02$ \\
WSM & 2 & $0.35 \pm 0.02$ & - & $0.03 \pm 0.01$ & $0.37 \pm 0.02$ \\
EN & 2 & $0.20 \pm 0.02$ & - & $0.04 \pm 0.01$ & $0.24 \pm 0.01$ \\
EW & 3 & $0.26 \pm 0.02$ & $0.03 \pm 0.01$ & - & $0.29 \pm 0.01$ \\
\hline
\end{tabular}

Model 1: model including direct genetic effects, Model 2: model including direct genetic and maternal permanent environmental effects, Model 3: model including direct and maternal genetic effects, Model 5: model including direct and maternal genetic effects and maternal permanent environmental effects 
Considerable differences were found in variance components and consequently in the heritability estimates across models. Maternal effects represented a source of variation for all traits except for ASM, although the genetic part of maternal effects was observed only for $\mathrm{BW}_{0^{\prime}} \mathrm{BW}_{8^{\prime}} \mathrm{BW}_{12}$ and $\mathrm{EW}$.

Estimated maternal heritability was moderate for $\mathrm{BW}_{0}(0.30)$ and low for $\mathrm{BW}_{8^{\prime}} \mathrm{BW}_{12}$ and EW (0.01, 0.02 and 0.03 , respectively). In the study of Le Bihan-Duval et al. (1998), the variance explained by the maternal effects accounted for a rather small part of the total phenotypic variance for BW at different ages in broilers (from $3 \%$ to $8 \%$ according to the trait).

Maternal genetic and maternal environmental effects in Mexico native fowl were reported to be $16 \%$ and $8 \%$, respectively (Prado-Gonzalez et al. 2003).

Maternal heritability and the proportion of permanent environmental variance to phenotypic variance for BW0 ( 0.30 and 0.25 , respectively) were higher than for BW at older ages (from 0.01 to 0.04 ), while its direct heritability (0.05) was lower than for BW at older ages (from 0.17 to 0.35 ). Similar results were reported by Prado-Gonzalez et al. (2003) in Mexico native fowl and Norris \& Ngambi (2006) in local Venda chickens. Hartmann et al. (2003) estimated direct and maternal genetic heritabilities for $\mathrm{BW}_{0}$ in a White Leghorn line and showed that direct heritability (0.01) was lower than maternal genetic heritability (0.5) for this trait. Dana et al. (2011) used an animal model consisting of direct genetic and common environmental effects for BW traits in Horro chicken of Ethiopia. The value of direct heritability for $\mathrm{BW}_{0}(0.40)$ in their study was higher than present results. This might be due to missing maternal genetic effects in their analyses. Saatci et al. (2006) found that BW ${ }_{0}$ had the lowest direct heritability and the highest maternal heritability among performance traits in Japanese quail that is in agreement with the results of the current study.

The maternal permanent environmental effects were not important for EW while maternal genetic effects were considerable. However, Saatci et al. (2006) reported that maternal effects are not important for EW in Japanese quail. The maternal genetic and environmental effects are not relevant for ASM. In contrast, Ghorbani et al. (2012) reported that these effects were significantly influenced by ASM. They also found that the model including genetic and environmental maternal effects was the best model for EN and WSM while present results indicated that only the environmental part of maternal effects was important for EN and WSM in Isfahan native fowl. The observed differences among studies might be due to breed differences, environmental conditions, number of observations and the statistical method of analysis.

Direct heritability estimates for $\mathrm{BW}_{8^{\prime}} \mathrm{BW}_{12^{\prime}} \mathrm{ASM}, \mathrm{WSM}, \mathrm{EN}$ and $\mathrm{EW}$ were moderate $(0.17$ to 0.35 ), which suggest a possible genetic improvement for these traits through selection.

Maternal genetic and permanent environmental effects play an important role in improvement of BW traits in this population. For WSM and EN, maternal permanent environmental effects were important, in addition to direct additive genetic effects. Therefore, maternal effects should be considered in genetic evaluations to obtain an accurate prediction of breeding values of the individuals, assuring more efficient selection. Clément et al. (2001) demonstrated that if maternal genetic effects existed but they were not considered in the model, the direct heritability coefficient was overestimated. Praharani (2009) stated that heritability estimates of growth traits in Bali cattle resulting from analysis without maternal effects were overestimated.

Inclusion of maternal (genetic and environmental) effects into the models caused a decrease in direct heritability estimates. 
Genetic, environmental and phenotypic trends were determined to evaluate the breeding program performed in Isfahan native fowl breeding centre. Maternal genetic trend was also studied through generations. The maternal genetic trend for economic traits in native fowl has not been reported in the literature. The values and the significance of trends are presented in Table 3.

Table 3

The values of direct genetic, maternal genetic, environmental and phenotypic trends with their standard errors for studied traits in Iranian native fowl

\begin{tabular}{lcccr}
\hline Trait & Direct genetic trend & Maternal genetic trend & Environmental trend & Phenotypic trend \\
\hline BW $_{0}$ & $-0.01 \pm 0.00^{* * *}$ & $0.03 \pm 0.00^{* * * *}$ & $-0.17 \pm 0.01^{* * *}$ & $-0.17 \pm 0.01^{* * *}$ \\
BW $_{8}$ & $7.99 \pm 0.03^{* * *}$ & $0.12 \pm 0.00^{* * *}$ & $16.72 \pm 0.19^{* * *}$ & $27.97 \pm 0.21^{* * *}$ \\
BW $_{12}$ & $9.96 \pm 0.04^{* * *}$ & $0.85 \pm 0.01^{* * *}$ & $8.01 \pm 0.23^{* * *}$ & $17.87 \pm 0.25^{* * *}$ \\
ASM & $-0.76 \pm 0.00^{* * *}$ & - & $0.45 \pm 0.02^{* * *}$ & $-0.27 \pm 0.02^{* * *}$ \\
WSM & $8.14 \pm 0.05^{* * *}$ & - & $0.05 \pm 0.256^{\text {ns }}$ & $7.51 \pm 0.31^{* * *}$ \\
EN & $0.79 \pm 0.00^{* * *}$ & - & $0.61 \pm 0.02^{* * *}$ & $1.37 \pm 0.02^{* * * *}$ \\
EW & $0.05 \pm 0.00^{* * *}$ & $0.01 \pm 0.00^{* * *}$ & $0.22 \pm 0.00^{* * *}$ & $0.27 \pm 0.01^{* * *}$ \\
\hline
\end{tabular}

$* * *: P<0.001$, ns: not significant

Direct and maternal genetic trends together with environmental and phenotypic trends were calculated based on the values obtained from the best model for each trait.

For $\mathrm{BW}_{0}$, the maternal genetic trend was low and positive, but the direct genetic trend was slightly negative. This can be due to negative correlation between direct and maternal genetic effects (results not shown). However, negative environmental conditions led to further exacerbation of the phenotypic trend for this trait.

Direct and maternal genetic trends for $\mathrm{BW}_{8}$ and $\mathrm{BW}_{12}$ were significantly positive. It is an evidence for existence of positive correlation between direct and maternal genetic effects for these traits. Convergence could not be achieved with the ASREML software if the correlation between these effects was included in the model. Positive direct genetic trends for BW 8 and $\mathrm{BW}_{12}$ showed that the performed breeding programs for these traits were successful. Although the maternal genetic trend was significant, it was of low importance for the improvement of these traits because of low maternal heritability. The phenotypic trends for these traits were also significantly positive. It means that environmental conditions together with genetic structure were improved through generations for these traits.

Direct genetic trends for WSM and EN were significantly positive, which shows that the performed breeding program for these traits was also successful. The phenotypic trend was close to the genetic trend for WSM because of a low environmental trend for this trait. There was a significantly negative direct genetic trend for ASM, which is favourable for this trait. The phenotypic trend was significant but weaker than the direct genetic trend because of the positive environmental trend for this trait.

For EW, the phenotypic trend was strongly positive, while direct and maternal genetic trends were slightly positive. It indicates that the phenotypic improvement was due to better environmental conditions rather than genetic improvement for this trait.

The average of direct and maternal breeding values and phenotypic values through generations for studied traits is presented in Figure 1. 


\section{Body Weight at hatch}

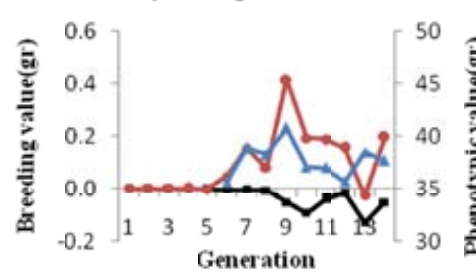

-Direct breeding value

$\rightarrow$ Maternal breeding value

—Phe notypic value

\section{Body Weight at 12 weeks of} age

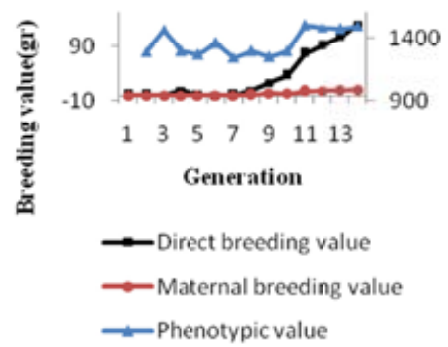

Weight at Sexual Matutity

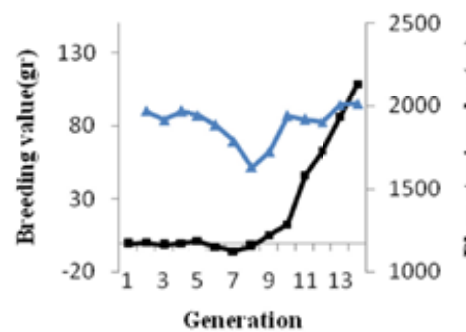

—-Breeding value $\longleftarrow$ Phenotypic value

\section{Egg Weight}

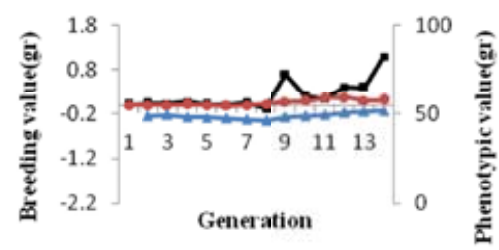

-Direct breeding value

—-Maternal breeding value

—-Phenotypic value
Body Weight at 8 weeks of

age

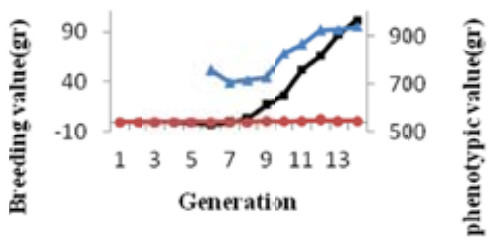

—Direct breeding value

- Maternal breeding value

-Phenotypic value

Age at Sexual Maturity

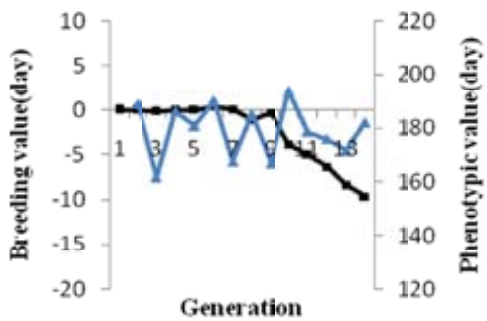

—Breeding value —-Phenotypic value

\section{Egg Number}

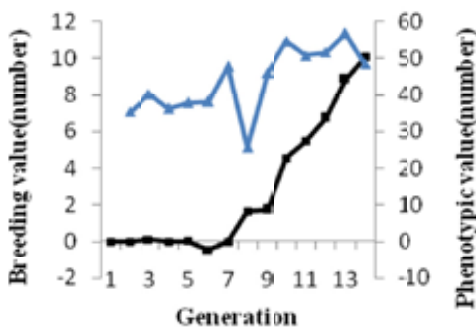

$\longrightarrow$ Breeding value $\longrightarrow$ Phenotypic value
Figure 1

The average of direct and maternal breeding values and phenotypic values of traits through generations 
Based on this figure, the phenotypic trend was in the same direction as the direct genetic trend for all traits except ASM. It was probably due to the positive and unfavourable environmental trend for this trait. Therefore, it is important to improve environmental conditions, e.g. the quality of nutrition, to allow birds to lay at younger ages. It causes higher egg production during the lifetime of birds. The direct breeding values were consistently increased through generations. On the other hand, for $\mathrm{BW}_{8^{\prime}} \mathrm{BW}_{12}, \mathrm{WSM}, \mathrm{EN}$ and $\mathrm{EW}$ the maternal genetic trend for $\mathrm{BW}_{8^{\prime}} \mathrm{BW}_{12}$ and $\mathrm{EW}$ also showed the same direction as the direct genetic trend but to a smaller extent. The breeding values for ASM were reduced through generations, showing that birds started to lay eggs at younger ages.

In conclusion, the present study indicated that economic traits were significantly influenced by maternal effects. For all traits except ASM, it was favourable to include additive genetic and maternal genetic and permanent environmental effects in the estimation of breeding values in the population of Isfahan native fowl.

Also, the trends of studied traits showed that the performed breeding program effectively improved the genetic structure of these traits.

\section{References}

Akaike H (1974) A New Look at the Statistical Model Identification. IEEE Trans Automat Contr AC-19, 716-723

Anderson TW, Darling DA (1952) Asymptotic Theory of Certain »Goodness of Fit« Criteria Based on Stochastic Processes. Ann Math Statist 23, 193-212

Clément V, Bibé B, Verrier E, Elsen JM, Manfredi E, Bouix J, Hanocq E (2001) Simulation analysis to test the influence of model adequacy and data structure on the estimation of genetic parameters for traits with direct and maternal effects. Genet Sel Evol 33, 369-395

Dana N, vander Waaij EH, van Arendonk JAM (2011) Genetic and phenotypic parameter estimates for body weights and egg production in Horro chicken of Ethiopia. Trop Anim Health Prod 43, 21-28

Emamgholi Begli H, Zerehdaran S, Hassani S, Abbasi MA, Khan Ahmadi AR (2010) Heritability, genetic and phenotypic correlations of egg quality traits in Iranian native fowl. Br Poult Sci 51, 740-744

Gilmour AR, Thompson R, Cullis BR, Welham SJ (2000) ASREML Reference Manual. New South Wales Agriculture, Orange, Australia

Ghorbani S, Kamali MA, Abbasi MA, Ghafouri-Kesbi F (2012) Estimation of Maternal Effects on Some Economic Traits of North Iranian Native Fowls Using Different Models. J Agr Sci Tech 14, 95-103

Grosso JLBM, Balieiro JCC, Eler JP, Ferraz JBS, Mattos EC, Michelan Filho T (2010) Comparison of different models to estimate genetic parameters for carcass traits in a commercial broiler line. Genet Mol Res 9, 908-918

Hartmann C, Johansson K, Strandberg E, Rydhmer L (2003) Genetic correlations between the maternal genetic effect on chick weight and the direct genetic effects on egg composition traits in White Leghorn line. Poult Sci 82, 1-8

Haunshi S, Shanmugam M, Padhi MK, Niranjan M, Rajkumar U, Reddy MR, Panda AK (2012) Evaluation of two Indian native chicken breeds for reproduction traits and heritability of juvenile growth traits. Trop Anim Health Prod 44, 969-973

Horst P (1989) Native fowl as reservoir for genomes and major genes with direct and indirect effects on the adaptability and their potential for tropically orientated breeding plans, Eur Poult Sci 53, 93-101

Kamali MA, Ghorbani SH, Moradi Sharbabak M, Zamiri MJ (2007) Heritabilities and genetic correlation of economic traits in Iranian native fowl and estimated genetic trend and inbreeding coefficients. Br Poult Sci 48, 443-448 
Kiani-Manesh HR (2000) Estimation of (co)variance components of economically important traits in Iranian native fowls. M.Sc.Thesis, Mazandaran University, Iran

Le Bihan-Duval E, Mignon-Grasteau S, Millet N, Beaumont C (1998) Genetic analysis of a selection experiment on increased body weight and breast muscle weight as well as on limited abdominal fat weight. Br Poult Sci 39, 346-353

Meyer K (1997) Estimates of genetic parameters for weaning weight of beef cattle accounting for directmaternal environmental covariances. Livest Prod Sci 52, 187-199

Norris D, Ngambi JW (2006) Genetic parameter estimates for body weight in local Venda chickens. Trop Anim Health Prod 38, 605-609

Prado-González EA, Ramirez-Avila L, Segura-Correa JC (2003) Genetic parameters for body weights of Creole chickens from Southeastern Mexico using an animal model. Livest Res Rural Dev 15, 1-7

Praharani L (2009) Estimation of direct and maternal effects for weaning and yearling weights in Bali cattle. Indones J Agric 2, 74-81

Saatci M, Omed H, Dewi, IA (2006) Genetic parameters from univariate and bivariate analyses of egg and weight traits in Japanese quail. Poult Sci 85, 185-190

Sang BD, Kong HS, Kim HK, Choi CH, Kim SD, Cho YM, Sang BC, Lee JH, Jeon GJ, Lee HK (2006) Estimation of genetic parameters for economic traits in Korean native chickens. Asian-Aust J Anim Sci 3, 319-323

SAS Institute Inc. (2001) SAS/STAT Software: Changes and Enhancements, Release 8.2., SAS Institute Inc., Cary, NC, USA 